\title{
FRAGWÜRDIGE ANAMNESE
}

\section{Tripper ohne Sex}

- Die Angaben von Jugendlichen zu ihrem Sexualverhalten sind nicht immer vertrauenswürdig. In einer amerikanischen Studie hatte jeder zehnte Jugendliche mit einer nachgewiesenen sexuell übertragbaren Krankheit behauptet, in den letzten zwölf Monaten sexuell abstinent gewesen zu sein. Für die Studie waren 14012 Jugendliche und junge Erwachsene im Durchschnittsalter von 21 Jahren befragt worden. Sie hatten außerdem Urinproben abgegeben, die auf Chlamydia trachomatis, Neisseria gonorrhoeae und Trichomonas vaginalis untersucht worden waren.

\section{KINDER UND IHR GAMEBOY}

\section{Daddeln bis es weh tut}

- Schmerzen in Finger- und Handgelenken sind die Folge, wenn Kinder und Jugendliche zu lange mit Spielgeräten wie dem Gameboy hantieren. Es besteht eine direkte Korrelation zwischen der Stärke der Schmerzen und der Spielzeit. Dies fanden Forscher des NYU Hospital for Joint Diseases, New York, in einer Studie mit 257 Kindern und Jugendlichen im Alter von neun bis 15 Jahren heraus. Spielen mit Gameboy und Xbox führte zu signifikant stärkeren Be-

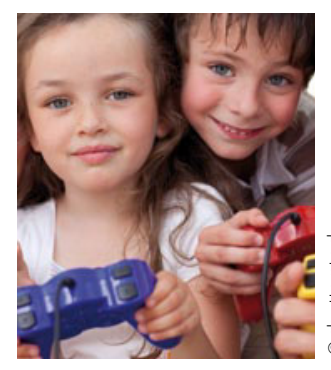

Macht's noch Spaß? schwerden als stundenlanges Hantieren mit dem Handy. Mädchen, so zeigte die Studie, klagten zweimal häufiger über Schmerzen als Jungen.

Vortrag bei der EULAR 2011, London Mai 2011

\section{DARMKREBSSCREENING BEI MÄNNERN}

\section{Mit 55 zu spät?}

— Seit 2002 können GK-Versicherte ab 55 Jahren zur Vorsorge-Koloskopie. Männer haben aber in jedem Alter ein deutlich höheres Risiko für fortgeschrittene Adenome und Tumoren des Dickdarms als Frauen, zeigt eine Studie der Universität München. Bei der Screening-Koloskopie wurden fortgeschrittene Adenome bei 5925 Frauen (5,3\%) und 7923 Männern (9,8\%) entdeckt,

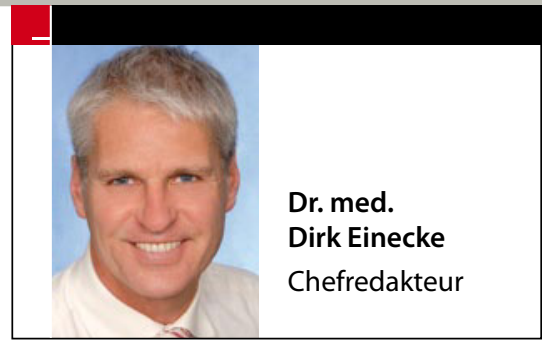

\section{ÜBERGEWICHT}

\section{Schlafentzug macht Kinder dick}

— Es gibt viele Gründe, weshalb es wichtig ist, dass Kinder ausreichend schlafen. Prophylaxe von Übergewicht zählt auch dazu. Wie eine Längsschnittstudie mit 244 Kindern im Alter zwischen drei und sieben Jahren ergab, führt jede zusätzliche Stunde Schlaf pro Nacht im Alter zwischen drei und fünf Jahren zu einer Verminderung des BMI um 0,48 im Alter von sieben Jahren. Dies entspricht bei durchschnittlicher Größe einem Gewichtsunterschied von 0,7 kg. Das Risiko, mit sieben Jahren übergewichtig zu sein, sank dadurch um $61 \%$.

BMI 2011;342:d2712

\section{Nächste Woche:}

\section{CME-Schwerpunktheft der MMW mit Jubiläum}

Hier kommt (Fortbildungs-) Freude auf: Im nächsten Heft (MMW 23/2011) erscheint die 300. Folge unserer Zertifizierten Fortbildung (CME). Das macht insgesamt 900 CME-Punkte, die Sie mit uns in den vergangenen zwölf Jahren sammeln konnten. Und weil uns Ihre Fortbildung auch weiterhin am Herzen liegt, haben wir für die Ausgabe 23 gleich ein Schwerpunktheft mit zehn praxisnahen CME-Beiträgen vorbereitet, u.a. zu folgenden Themen: Impfen, Juckreiz, Badeotitis, Praktische Insulintherapie, Akuter Thoraxschmerz ... (Teilnahme ab 9.6. unter www.cme-punkt.de!) 